\title{
GLIOBLASTOMA MULTIFORME CEREBELAR
}

\author{
RELATO DE UM CASO EM CRIANCA
}

\author{
R. РОМВО * \\ A. TORTELLY-COSTA ** \\ Fु. BULACIO *** \\ M. D. HAHN ** \\ M. L. DE CARVALHO **
}

Gliobastomas multiformes (GM) representam cerca de $50 \%$ das neoplasias do sistema nervoso central (SNC), menos de $3 \%$ ocorrendo na infância. 4,5. Este número significa em torno de $7 \%$ dos tumores intracranianos da criança 5. Os GM podem surgir em qualquer região do SNC, todavia a sua localização na fossa posterior é relatada como rara, tendo no cerebelo uma situação excepcional 1,2,3,4,5,6,7,8,11,13. Existe grande tendência nos tumores da infância para exibir aspecto histológico de características bizarras, algumas vezes dificultando sua classificação histológica definitiva ${ }^{4}$.

O presente relato de caso de GM no cerebelo, parece tratar-se do segundo referido na literatura nacional em criança ${ }^{3}$. É propósito também rever os casos publicados e tecer considerações sobre a dificuldade diagnóstica histopatológica deste tumor, confrontado com as outras neoplasias gliais dessa região.

\section{OBSERVAÇÃO}

L.A.F., paciente branca, com 9 anos de idade, registro 287.097, acimitida no Hospital Universitário Antonio Pedro em 25-02-83, encaminhada do interior do Estado do Rio de Janeiro por apresentar paralisia facial periférica direita, síndrome cerebelar ipsolateral e confusão mental. O quadro se iniciara há 7 dias. Exame neurológico - criança calma, falando, algo desorientada halo e autopsiquicamente, incapaz de manter-se sentada. Pares cranianos: hipoestesia corneana à direita e paralisia do reto externo bilateralmente. Hemiparesia acentuada à direita com os reflexos profundos diminuídos em relação à esquerda. Ausência de sinal de Babinski e equivalentes. Coordenação conservada à esquerda. Fundo de olho: edema de papila óptica incipiente, com ausência de pulso venoso. Marcha atáxica. Nistagmo horizontal em ambas as miradas laterais. Exame físico - Criança eupneica, apirética e hidratada, mucosas normocoradas, acianótica e anictérica; orofaringe com vestíbulo oral úmido, sem desvio de úvula; ausculta pulmonar

Trabalho realizado na Disciplina de Neurocirurgia (* Professor Assistente, ** Professor Adjunto, *** Professor Titular) e na Disciplina de Patologia (** Professor Adjunto) da Universidade Federal Fluminense. 
mostrando murmúrio vesicular presente sem ruídos adventícios; o exame do aparelho cardiovascular revelava um ritmo regular em dois tempos, bulhas normofonéticas, sem sopros; abdômen flácido e sem visceromegalias; membros sem edemas. Exames complementares - hemograma com normocitose, hematimetria e plaquetometria normais; perfil hidroeletrolítico dentro dos padrões de normalidade; elementos anormais na urina ausentes; coagulograma normal. Radiografia de crânio sem evidências de sinais de hipertensão intracraniana. Tomografia computadorizada de crânio revelando preser.ça de lesão expansiva de fossa posterior à direita (Fig. 1). Cirurgia - submetida a craniectomia de fossa posterior à direita que revelou a presença do lesão tumoral de cor acizentada, consistência gelatinosa, mal delimitada do parênquima cerebelar circunvizinho, bastante vascularizado, sem cavidades císticas. Retirada anostra para exame histopatológico sendo o restante removido por aspiração em sua maicr parte; a paciente permaneceu em coma no pós operatório, evoluindo para o óbito no décimo terceiro dia da cirurgia. Biópsia - $\mathrm{O}$ exame do material de biópsia mostrou à microscopia vários fragmentos de tecido cerebelar exibindo neoplasia da série glial ccinstituida de grande número de células extremamente atípicas, volumosas e pleomórficas, apresentando núcleos ora hipercromáticos, ora vesiculosos, com frequentes binucleações ou multinucleações. O citoplasma era algo abundante e eosinofílico. Tais células podiam ser observadas de forma isolada invadindo a camada granulosa e a molecular, ou entäo dispostas ao redor das estruturas vasculares. Notavam-se ainda extensas áreas de necrose, hemorragia e vasos com as luzes trombosadas (Fig. 2). Necropsia - $O$ estudo do SNC revelou na macroscopia cérebro pesando $1.050 \mathrm{~g}$, recoberto por leptomeninge discretamente espessada e edema moderado. Aos cortes observava-se discreta dilatação dos cornos posteriores de ambos os ventrículos laterais. O cerebelo apresentava extensa tumoração irregular, comprometendo todo o hemisfério direito, medindo $7,5 \times 4,5 \mathrm{~cm}$, de aspecto pardo acinzentado, friável, com áreas hemorrágicas (Fig. 3). Na microscopia

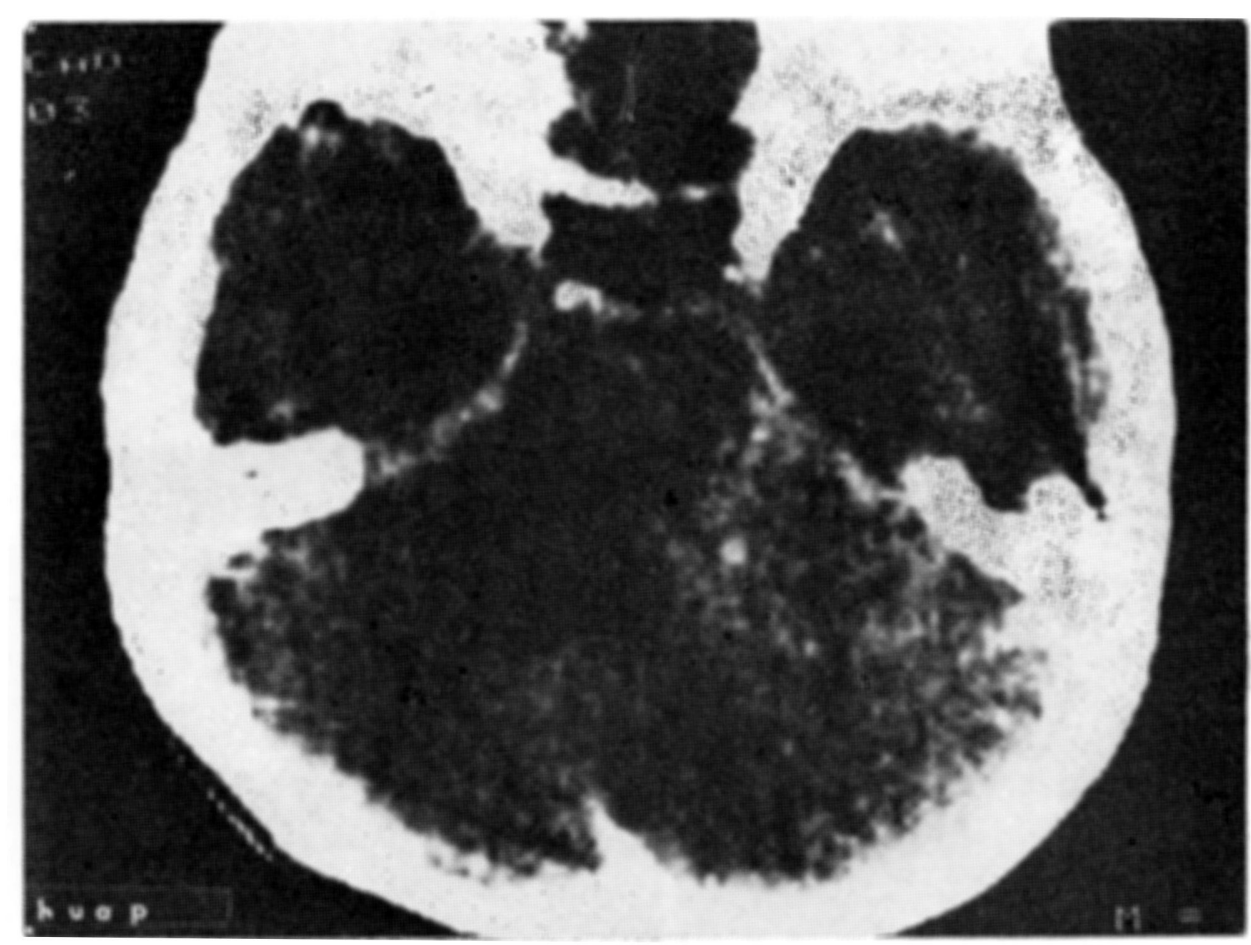

Fig. 1 - Caso L.A.F.: Tomografia computadorizada mostrando lesão expansiva no hemisfério cerebelar direito. 

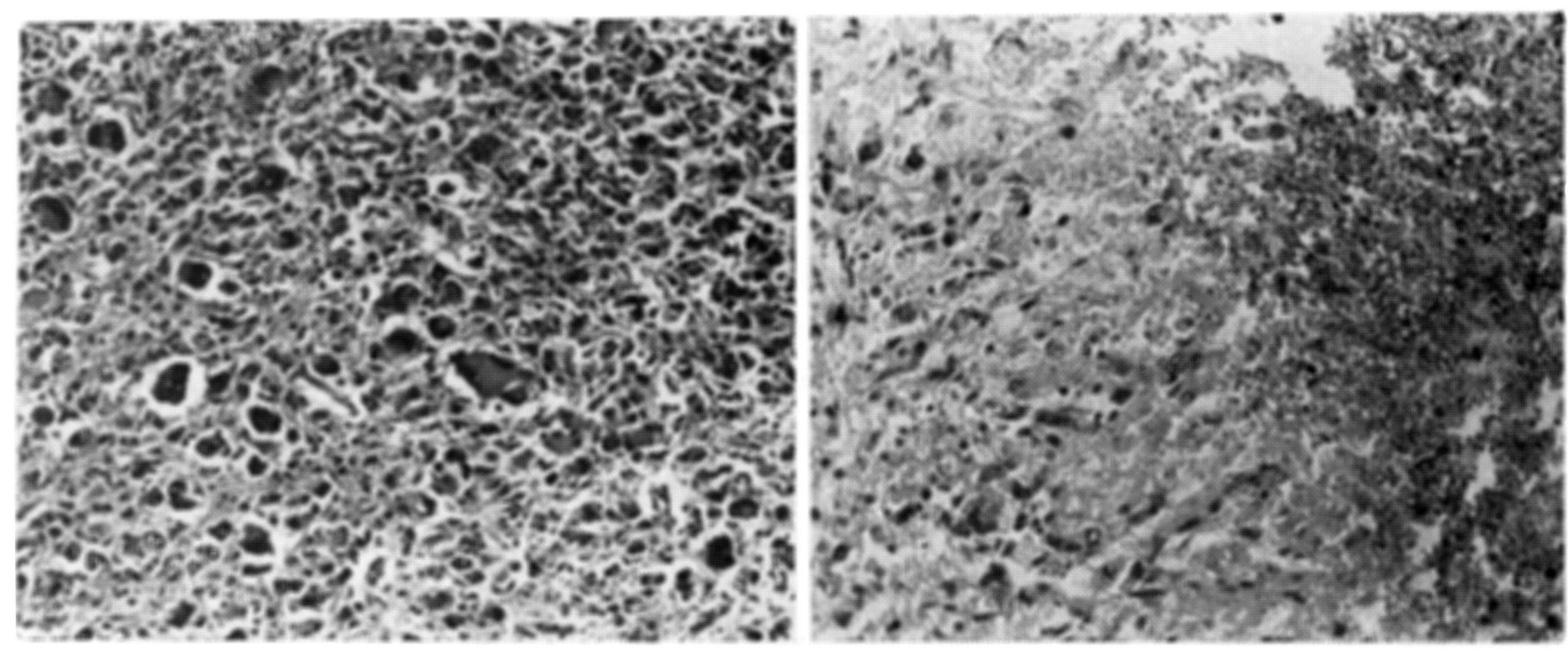

Fig. 2 - Caso L.A.F.: a esquerda, aspecto geral do tumor, cujas células mostram marcado pleomorfismo, com numerosas cellulas gigantes (H.E.; 150 X); a direita ärea de necrose com pseudo palicada, tipica do glioblastoma multiforme (H.E.; 60 X).

os cortes de cerebelo eram representados por neoplasia da série glial, com os mesmus achados descritos na biópsia, notando-se comprometimento do tecido pontino por células tumorais e invasão do espaço subaracnóideo. Os cortes efetuados a nível de hemisfério cerebral exibiam no espaço subaracnóideo a presença de moderado afluxo inflamatório polimorfonuclear neutrófilo. No parênquima havia acentuada dilatação dos espaços perivasculares, os quais continham áreas com células inflamatórias ncutrofilicas, aspecto este igualmente observado nos núcleos da base. O estudo dos cemais órgãos revelou, no pulmão, broncopneumonia extensa com áreas abscedadas. A causa mortis foi atribuída ao edema cerebral com herniação de amigada cercbelar direita e ì lesão pontina em consequência da presença do tumor.
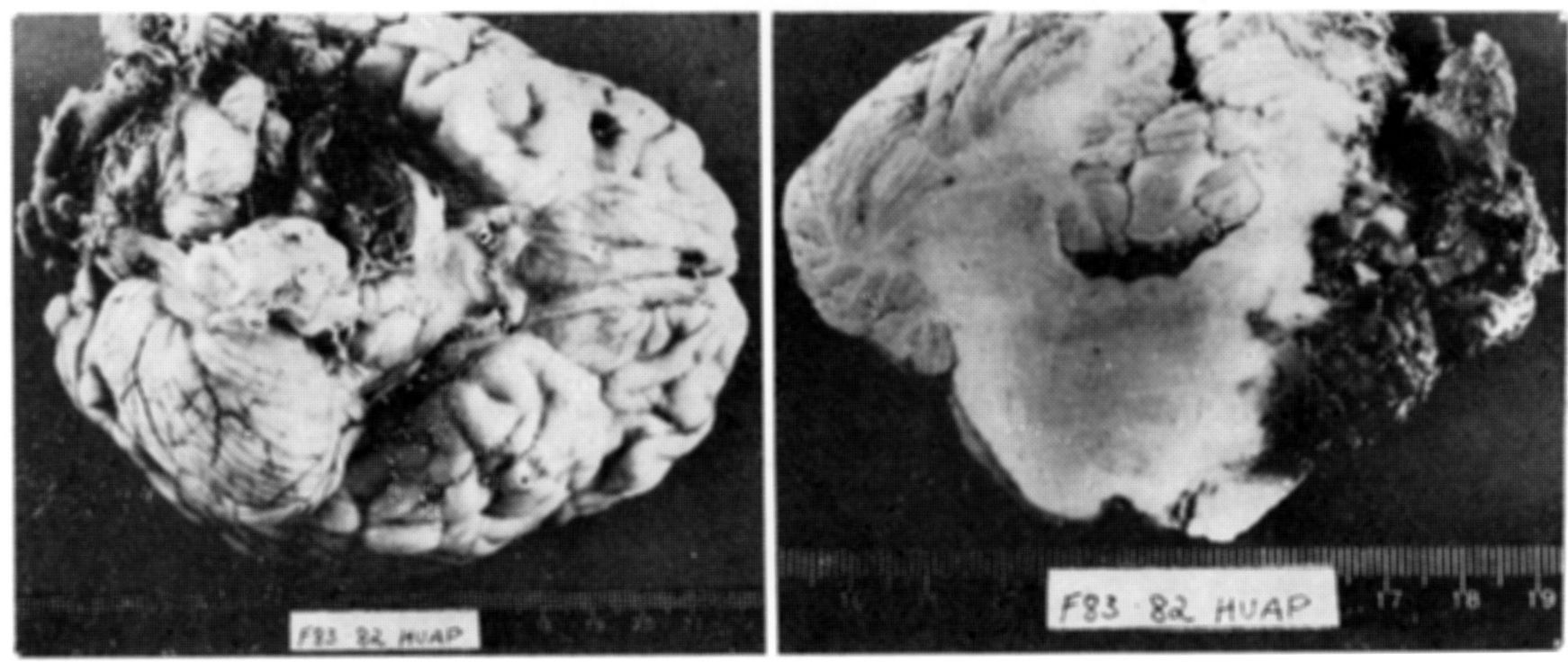

Fíg. 3 - Caso L.A.F.: à esquerdia, aspecto ventral do cérebro e cerebelo; à direita, corte das estruturas da fossa posterior, notando-se a invasião da ponte. 


\section{COMENTARIOS}

Glioblastoma multiforme foi o termo proposto por Bailey, em 1927, em substituição à denominação espongioblastoma multiforme que Globus e Strauss utilizaram 2;4, em 1925, para referir tipo de tumor da série glial, de crescimento rápido. Esses autores acreditavam que este tumor, uma das formas mais anaplásicas das neoplasias primárias intracranianas, era composto de células primitivas embrionárias 11,13. Atualmente alguns autores consideram que o GM se desenvolve por desdiferenciação de parte de um tumor maduro, predominantemente de origem astrocitária ${ }^{1,2,13}$, dado que alterações gliomatosas são encontradas em astrocitomas pontinos em crianças ${ }^{13}$. A despeito de Kernohan e Sayre haverem abandonado o termo gliobastoma multiforme e substituido por astrocitoma grau III e IV, a denominação ainda encontra aceitação ${ }^{2,13}$ sobretudo naqueles casos nos quais proveniência astrocitária não é plenamente evidente 13. As variações histológicas observadas microscopicamente nos GM, com celularidade ora uniforme ora com células extremamente pleomórficas em estágios intermediários, poderão impedir o reconhecimento desta neoplasia. Assim, autores há que têm proposto que esses tumores sejam denominados gliomas indiferenciados, numa tentativa de caracterizar sua evolução maligna, indiferentes a sua fase inicial, dada a rápida progressão de suas características anaplásicas ${ }^{12}$.

Ao revermos a literatura encontramos referência textual de glioblastoma multiforme no cerebelo em 40 casos desde 1928, sendo o primeiro de Carmichael e o último de Cardoso e col. ${ }^{3}$, em 1983. Na literatura brasileira, além do cáso de Cardoso, foi registrada a presença de GM em cerebelo por Aidar Aun e col., sendo este o caso de uma mulher de 57 anos ${ }^{1}$. Em cerca de 60 anos o relato deste pequeno número de casos parece indicar que a patologia é de real raridade, ressalvando-se ainda que em 10 casos não foram publicados os achados histológicos. A despeito do número aparentemente reduzido de referências do GM no cerebelo, encontramos algumas anotações que julgamos pertinente analisar. Assim, Ingraham e Matson, em 418 tumores de fossa posterior em crianças e adolescentes, acusam a presença de "raros astrocitomas graus III e IV" nessa região, cujo tipo foi reconhecido apenas ao exame microscópico ${ }^{10}$. Os autores não precisaram o número desses tumores nem a sua nítida topografia $\mathrm{e}$, por este motivo, provavelmente não foram catalogados em levantamentos posteriores. Kopelson refere 8 casos de GM cerebelar, classificando as lesões do tipo astrocitomas grau III e IV como glioblastomas, apesar de assinalar tendência adotada por autores no sentido de apenas os graus avançados de l'sões anaplásicas gliais deverem ser classificados como tal ${ }^{9}$. Em 1932, Bailey $\dot{e}$ Eisenhardt consideraram a existência de 32 exemplos de espongioblastoma intracraniano, entre os quais 7 de cerebelo 4 . Tendo em vista as indefinições anátomo-patológicas conccituais e a precariedade técnico-instrumental naquela época, inquirimos se não seriam aqueles 7 espongioblastomas enquadráveis hoje no diagnóstico de GM. Levando em conta essas considerações sobre controvérsias de classificação histológica, a incidência dos GM no cerebelo elevar-se-ia paia em torno de 60 casos 3,4,5,6,7,8. Assim, consideramos ser o GM cerebelar mais frequente do que se crê, a julgar pelos relatos específicos desse tumor nesse sítio do SNC, em 
virtude das divergências existentes sobre a correta definição anátomo-patológica dessa neoplasia. A despeito desta ponderação sobre a presunção quanto a ser maior do que se supõe a incidência dos GM no cerebelo, sustentamos que esta patologia permanece rara.

Rubinstein relaciona ordem de incidência dos GM no lobo frontal, lobo temporal e corpo caloso e outras estruturas medianas ${ }^{13}$, sendo estas últimas topografias questionadas por Schmideck \& Sweet 14. Estes autores concordam com a prevalência supratentorial $(97,9 \%)$ dos GM e distribuem sua incidência por ordem: lobo frontal $(36,7 \%)$, lobo temporal (33,9\%), lobo parietal (25\%) e lobo occipital (2,2\%). Ponderam que o comprometimento das estruturas medianas é observada porque, por ocasião dos diagnósticos desses tumores, elas já estão envolvidas por extensão, pelas comissuras e tractos dentro da substância branca. Verificamos assim que restam tão somente $2,2 \%$ de localização do GM para toda a fossa posterior 14. Astrocitomas pontinos mostram, em $60 \%$ dos casos, evidência de malignidade; em contraste, menos de $10 \%$ dess astrocitomas de cerebelo revelam características malignas ${ }^{13}$. Esses fatos referendam a raridade da patologia referida neste relato, ressalvando todavia que a incidência encointrada em revisões de casos, comparadas com referências bibliográficas, deverá ser maior que a conhecida.

\section{RESUMO}

Os autores relatam o segundo caso publicado no Brasıl de glioblastoma cerebelar na infância. A despeito da escassa citação na literatura mundial, questionam a raridade real desta patologia, achando que deverá ser mais frequente do que é acreditado, tendo em vista as indefinições e controvérsias anátomo-patológicas desta neoplasia.

\section{SUMMARY}

Glioblastoma multiforme in the cerebellum: report of one case in a child.

The authors relate a case of glioblastoma multiforme of the cerebellum in a nine-years old girl. They correlate this case with reports published and they think that this pathology is not so rare as believed. This is the second case report, in Brazil, in children.

\section{REFERENCIAS}

1. AIDAR AUN, R.; STAVALE, J.N. \& SILVA Jr., D. - Glioblastoma multiforme no cerebelo. Registro de um caso. Arq. Neuro-Psiquiat. (São Pạulo) 39:350, 1981.

2. BELL, W.E. \& MCCORMICK, W.F. - Increased Intracranial Pressure in Children. Saunders, Philadelphia, 1972.

3. CARDOSO, S.H.; STAVALE, J.N.; PEREIRA Fo, O.D.; BUENO, A.R. \& JACKSON, S.M. - Glioblastoma multiforme no cerebelo. Relato de um caso em criança. J. Pediat. 54:24, 1983. 
4. CUNEO, H. \& RAND, C.W. - Tumores Cerebrales en la Infância. Alfa, Buenos Ayres, 1955, pg. 88.

5. DOHRMANN, G.J.; FARWELL, J.R. \& FLENNERY, B.S. - Glicblastoma multiforme in children. J. Neurosurg. 44:442, 1976.

6. FRESH, C.B. \& O'BRIEN, M.S. - Cerebellar glioblastoma in children. J. Neurosurg. 45:705, 1976.

7. GRANT, F.C. \& JONES, R.K. - A clinical study of two hundred posterior fossa glioma in children. Clin. Neurosurg. $5: 1,1958$.

8. KATSURA, S.; SUSUKI, J. \& WADA, T. - A statistical study of brain tumors in the neurosurgical clinics in Japan. J. Neurosurg. 16:570, 1959.

9. KOPELSON, G. - Cerebellar glioblastoma. Cancer 50:308, 1982.

10. MATSON, D. - Neurosurgery of Infancy and Childhood. Ed.2. Charles C. Thomas, Springfield, 1969, pg. 410.

11. REED, U.C. - Tumores raros de fossa posterior na infância. Arq. bras. Neurocir. $1: 127,1982$.

12. ROSAI, J. - Ackerman's Surgical Pathology. Ed. G. Mosby, St. Louis, 1981, vol. II, pg. 1568.

13. RUBINSTEIN, L.J. - Tumours of the Central Nervous System. Armed Forces Institute of Pathology, Washington, 1970.

14. SCHMIDEK, H. \& SWEET, W. - Operative Neurosurgical Techniques. Grune \& Stratton, New York, 1982, vol. I, pg. 448.

Hospital Universitário Antonio Pedro - Rua Marques do Paraná - 24000, Niterói, RJ - Brasil. 